\title{
On the Lengths, Colours and Ages of Bars
}

\author{
Dimitri A. Gadotti ${ }^{1}$ and Ronaldo E. de Souza ${ }^{2}$ \\ ${ }^{1}$ Max-Planck-Institut für Astrophysik, Karl-Schwarzschild-Str. 1, D-85741, Garching bei \\ München, Germany \\ email: dimitri@mpa-garching.mpg.de \\ ${ }^{2}$ Departamento de Astronomia, Universidade de São Paulo, Rua do Matão, 1226, 05508-090, \\ São Paulo-SP, Brazil
}

\begin{abstract}
In an effort to obtain further observational evidences for secular evolution processes in galaxies, as well as observational constraints to current theoretical models of secular evolution, we have used BVRI and Ks images of a sample of 18 barred galaxies to measure the lengths and colours of bars, create colour maps and estimate global colour gradients. In addition, applying a method we developed in a previous article, we could distinguish for 7 galaxies in our sample those whose bars have been recently formed from the ones with already evolved bars. We estimated an average difference in the optical colours between young and evolved bars that may be translated to an age difference of the order of $10 \mathrm{Gyr}$, meaning that bars may be long standing structures. Moreover, our results show that, on average, evolved bars are longer than young bars. This seems to indicate that, during its evolution, a bar grows longer by capturing stars from the disk, in agreement with recent numerical and analytical results.
\end{abstract}

Keywords. Galaxies: evolution, galaxies: photometry, galaxies: structure

\section{Results and Implications}

The B-V colour difference between young and evolved bars is 0.4 mag, which can be translated to an age difference of $10 \mathrm{Gyr}$. This means that bars can be robust structures, in agreement with recent $n$-body simulations and observations of barred galaxies at higher redshifts. The young bars in our sample have an average length of $5.4 \pm 1.6 \mathrm{Kpc}$, while the evolved bars have an average length of $7.5 \pm 1.2 \mathrm{Kpc}$, consistent with recent theoretical expectations that bars grow longer while aging. Young bars are preferentially found in late-type spirals, indicating that bar recurrence may be more frequent in gas-rich, diskdominated galaxies. We also found that AGN are preferentially hosted by galaxies with young bars, suggesting that the fueling of AGN by bars happens in short timescales and that a clearer bar-AGN connection would be found in a sample of galaxies with young bars. We have also found that bar colours might be used as a proxy for bar ages. Enlarging the sample of bars with measured ages is paramount to calibrate this relation, confirm these results, compare in more detail observations and models, and better understand secular evolution. See Gadotti \& de Souza (2005, 2006) for further details.

\section{Acknowledgements}

DAG would like to thank the Deutsche Forschungsgemeinschaft and the Max-PlanckGesellschaft for financial support.

\section{References}

Gadotti, D. A. \& de Souza, R. E. 2005, ApJ 629, 797.

Gadotti, D. A. \& de Souza, R. E. 2006, ApJS 163, 270. 